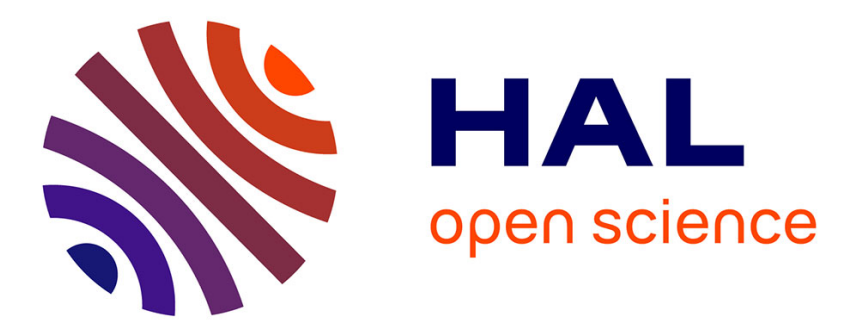

\title{
Can expertise modulate representational momentum?
}

Colin Blattler, Vincent Ferrari, André Didierjean, Pierre van Elslande, Evelyne Marmèche

\section{To cite this version:}

Colin Blattler, Vincent Ferrari, André Didierjean, Pierre van Elslande, Evelyne Marmèche. Can expertise modulate representational momentum?. Visual Cognition, 2014, 18, pp.1253 - 1273. 10.1080/13506281003737119 . hal-03347306

\section{HAL Id: hal-03347306 \\ https://hal.science/hal-03347306}

Submitted on 17 Sep 2021

HAL is a multi-disciplinary open access archive for the deposit and dissemination of scientific research documents, whether they are published or not. The documents may come from teaching and research institutions in France or abroad, or from public or private research centers.
L'archive ouverte pluridisciplinaire HAL, est destinée au dépôt et à la diffusion de documents scientifiques de niveau recherche, publiés ou non, émanant des établissements d'enseignement et de recherche français ou étrangers, des laboratoires publics ou privés. 
See discussions, stats, and author profiles for this publication at: https://www.researchgate.net/publication/228645604

\section{Can expertise modulate representational momentum?}

Article in Visual Cognition · April 2013

DOI: 10.1080/13506281003737119

CITATIONS

21

2 authors, including:

Colin Blättler

École de l'Air

19 PUBLICATIONS 106 CITATIONS

SEE PROFILE

Some of the authors of this publication are also working on these related projects:

Project interpersonal trust in teams (especially military teams whose members do not know each other) View project
READS

212 


\title{
Can Expertise Modulate Representational Momentum?
}

\author{
Colin Blättler \\ Laboratoire de Psychologie Cognitive, Université de Provence, Marseille, France

\section{Vincent Ferrari} \\ Centre de Recherche en Psychologie de la Connaissance, du Langage et de l'Émotion, \\ Université de Provence, Aix-en-Provence, France
}

André Didierjean

Université de Franche-Comté, Besançon, France

Pierre Van Elslande

Institut National de Recherche sur les Transports et leur Sécurité, Salon-de-Provence, France

Evelyne Marmèche

CNRS, Laboratoire de Psychologie Cognitive, Marseille, France

Short title: $\quad$ RM and expertise

Please address correspondence to: Colin Blattler, L.P.C. Université de Provence UMR 6146 Pôle 3C, Bâtiment 9 Case D3, Place Victor Hugo, 13331 Marseille Cedex 3, France. Email: colin.blattler@etu.univ-provence.fr Phone: +33 4885769 08. Fax: +33 (0)4 88576895

Keywords: Representational momentum; expertise; natural scenes. 


\begin{abstract}
Representational momentum (RM) refers to the tendency of participants to "remember" the stopping point of an event as being farther along in the direction of movement than it was in reality (Freyd \& Finke, 1984). Our aim was twofold: (1) test for the impact of domain-specific expertise (here, automobile driving) on RM, using films of road scenes, and (2) find out whether the improved anticipation ability that comes with greater expertise is transferred to scenes from domains that are far-removed from the person's domain of expertise. Two experiments were conducted in which experienced and inexperienced automobile drivers performed a movement-anticipation task on realistic road scenes (Experiment 1), with stimuli that were very different from those found in their domain of expertise (Experiment 2). These studies pointed out some properties of representational momentum, and showed that RM is dependent upon knowledge acquired by participants in specific domains. Our research also showed that expertise in automobile driving can modulate $\mathrm{RM}$ in road-scene perception (i.e, the cognitive characteristics of the observer can modulate the magnitude of the RM effect) but that expertise in automobile driving is not transferred to dissimilar domains.
\end{abstract}




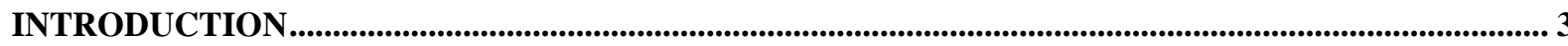

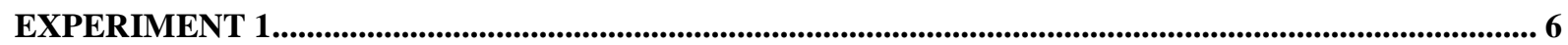

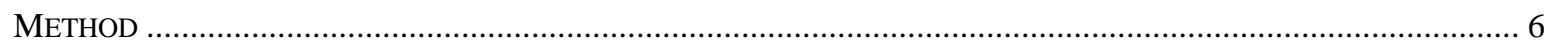

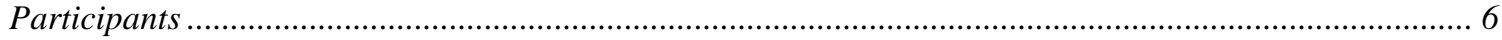

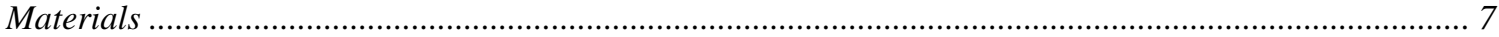

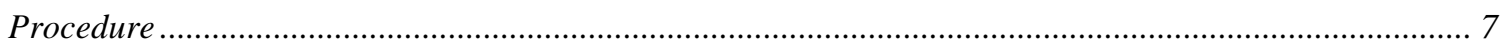

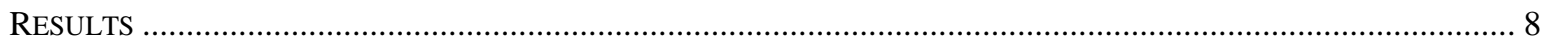

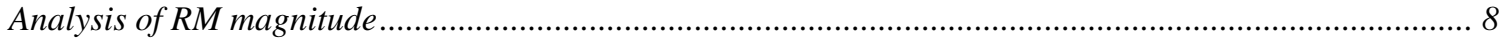

Analysis of the normal-resumption condition.................................................................................... 9

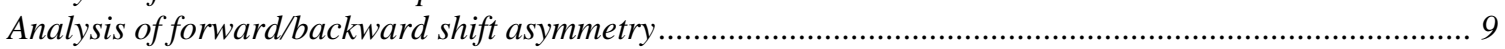

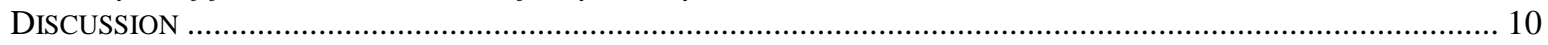

EXPERIMENT 2 ............................................................................................................................................. 11

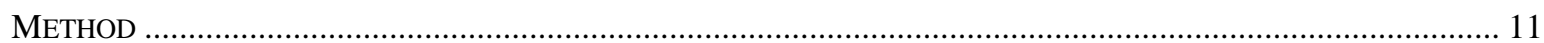

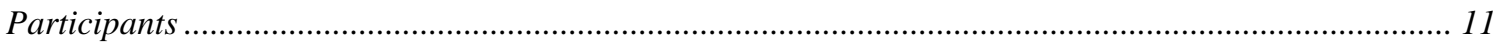

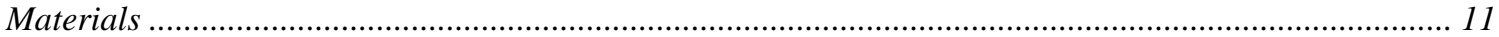

Procedure ……ㄴ..

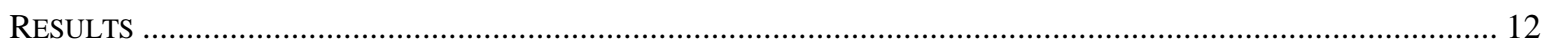

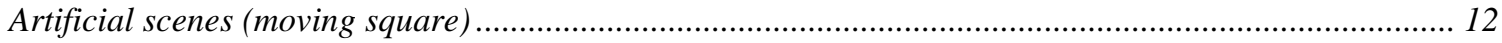

Natural scenes (person running) ................................................................................................. 13

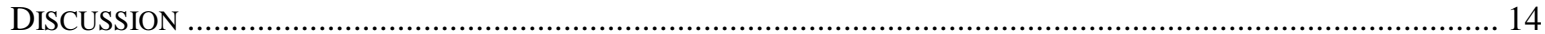

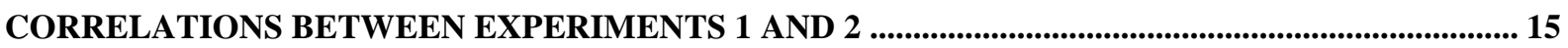

GENERAL DISCUSSION........................................................................................................................ 15

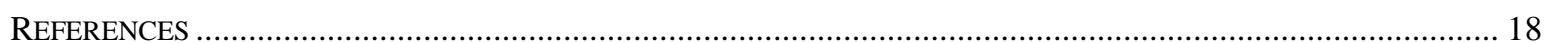

\section{Introduction}

Representational momentum refers to the tendency of participants to "remember" the stopping point of an event as being farther along in the direction of movement than it was in reality (Freyd \& Finke, 1984). For about 30 years now, this effect has been demonstrated using a wide variety of materials, with both dynamic stimuli (e.g. a moving dot, a rotating rectangle, the continuous motion of a set of dots; for a detailed review, see Hubbard, 2005) and static stimuli (drawings, still photographs of actions; Freyd, 1983; Freyd, Pantzer, \& Cheng, 1988). One of the conclusions drawn in all of these studies is that "frozen" actions are usually be perceived in terms of their dynamic dimension. This effect is robust. For example, if during the experimental phase, feedback about response accuracy is given to participants, this does not reduce RM (Ruppel, Fleming, \& Hubbard, 2009). Courtney and Hubbard (2008) 
even showed that the participants' knowing that the RM effect exists, or their wanting to counteract it, reduces the RM effect but does not eliminate it. Since the original work by Freyd and Finke (1984), a large number of studies on representational momentum (RM) have shown that when the cognitive system is processing a dynamic event, it has the ability to extrapolate the probable evolution of the current scene. Most of this research deals with the role played by the properties of a moving object in the RM effect, and to a lesser extent, how this effect is modulated by the perceiver's knowledge of the object. Some studies have shown that RM is modulated by the moving target's physical characteristics, such as its shape (Kelly \& Freyd, 1987; Nagaï \& Yagi, 2001), direction (Halpern \& Kelly, 1993; Hubbard, 1990; Munger, Solberg, Horrocks, \& Preston, 1999), speed (Freyd \& Finke, 1985), acceleration (Finke, Freyd, \& Shyi, 1986), and also whether it is moving away from or coming toward the participants (Hayes, Sacher, Thornton, Sereno, et Freyd ,1996; Hubbard ,1996), all of which can act as cues to where the object is likely to be located in the future.

Other studies have addressed RM by examining the effects of the observer's prior implicit knowledge, including the principles of physics such as gravity (Hubbard, 1995; Hubbard, \& Bharucha, 1988) and friction (Bertamini, 1993; Hubbard, 1998). The effect of declarative knowledge has also been studied. Finke and Shyi (1988) showed that the attempt to anticipate motion in an RM task does not make the RM effect any stronger. Kozhevnikov and Hegarty (2001) showed that experts in the laws of physics (e.g., the law of gravity) are not better at determining an object's spatial location in an RM task. Their studies showed that declarative knowledge does not modulate RM. Yet Vinson and Reed (2002) (see also Reed \& Vinson, 1996) showed that when inducing the upward movement of an object, if the object is said to be a rocket, the RM effect is stronger than when the object is said to be a building. In one case (the rocket), the object is associated with a feature that allows it to move upwards whereas in the other case (building), the object is not associated with such a feature. The authors thus showed that declarative knowledge can influence the processes underlying RM.

The study reported here falls in line with this research trend, insofar as it looks at the impact of the participant's level of expertise - here, in automobile driving - on RM. Although most studies on the RM effect have used relatively simple dynamic stimuli (a small number of items that are not action-related), a study by Thornton and Hayes (2004) (see also DeLucia \& Maldia, 2006; Munger, Owens, \& Conway, 2005) extended this effect to dynamic complex situations (videos depicting a synthesized image of a road as seen from inside a car driving at 55,65 , or $72 \mathrm{~km} / \mathrm{hr}$ ). In their experiment, participants viewed films of road scenes temporarily interrupted by a black screen lasting $250 \mathrm{~ms}$. After the interruption, the film continued and the participants had to judge whether the scene resumed at exactly the same point as it had 
stopped (normal-resumption condition) or at some other point. When the scene resumed at a different point, it could either be a shift forward (as if the car had quickly accelerated during the cut) or a shift backward (as if the car had backed up during the cut). The results obtained showed that forward shifts were more difficult to reject than backward shifts, and that the point deemed to be the most acceptable resumption point was shifted by about one meter in the car's direction of movement. This study thus showed that RM can also be found in the case of dynamic scenes.

The present study extends Thornton and Hayes's (2004) research. Our aim was twofold: (1) test for the impact of domain-specific expertise (here, automobile driving) on RM, using films of road scenes, and (2) find out whether the improved anticipation ability that comes with greater expertise is transferred to scenes from domains that are far-removed from the individual's domain of expertise.

In Experiment 1, experienced automobile drivers and inexperienced automobile drivers performed a movement-anticipation task on realistic road scenes (i.e., automobile driving filmed by an on-board camera). Many studies on expertise have shown that experts making judgments in their domain of expertise anticipate more than novices do, due to the large amount of domain-specific knowledge they have stored in long-term memory (Didierjean \& Marmèche, 2005; Doane, Sohn, \& Jodlowski, 2004; Ericsson \& Kintsch, 1995; Ferrari, Didierjean, \& Marmèche, 2006; for a review, see Didierjean \& Gobet, 2008). Our idea was to use an RM task similar to Thornton and Hayes's (2004) in order to find out whether an expertise effect occurs as early as the perceptual encoding phase. The fact of observing or not observing an expertise effect in the RM task should tell us whether or not some of the mechanisms responsible for this effect might be generic (i.e., general or not domain specific).

In Experiment 2, experienced drivers and inexperienced drivers performed two RM tasks with stimuli not involved in driving. One task showed a black square moving from left to right across the screen; the other showed a film of a person running. Our goal was to find out if the knowledge mobilized in RM tasks is partly task-specific. Most studies on cognitive expertise have shown that expert knowledge is not transferred to material that is not from the expert's domain (e.g., Chase \& Simon, 1973; Ericsson, 1985; Hatano \& Osawa, 1983; Unterrainer, Kaller, Halsband, \& Rahm, 2006; see however Gauthier, Williams, Tarr, \& Tanaka, 1998; Tanaka, Curran, \& Sheinberg, 2005). The results of Experiment 2 should tell us whether the anticipatory processes implemented by participants in RM tasks are solely domain-specific or whether they are transferable to other domains. 


\section{Experiment 1}

The purpose of this experiment was to study the role of experienced drivers' knowledge of automobile driving in their ability to anticipate motion in road scenes. The participants were divided into two groups (experienced drivers and inexperienced drivers) on the basis of their driving experience. They viewed road scenes filmed by an on-board camera. The scenes were interrupted by the display of a black screen lasting $250 \mathrm{~ms}$, and then resumed in one of three conditions: a shift forward (with respect to the car's direction of movement), a shift backward (in the direction opposite to the car's movement), or no shift (at exactly the same point as before the interruption (normal-resumption condition). In the shift conditions, the size of the forward and backward shifts was manipulated ( $\pm 3 \mathrm{~m}, \pm 6 \mathrm{~m}, \pm 9 \mathrm{~m}$, and $\pm 12 \mathrm{~m}$ ). The task was a same/different comparison task. Participants had to compare the last scene viewed before the cut, to the first scene viewed after the cut, and decide whether or not the two scenes were the same (i.e., whether the vehicle was in the same location in both scenes).

If more RM effect is observed in experienced drivers than inexperienced ones, then in the normal-resumption condition, experienced drivers should make significantly more errors than inexperienced ones. In the forward-shift and backward-shift conditions, if participants anticipate, they should have more trouble deciding on forward shifts (than on backward shifts) whether or not the first image seen after the cut is different from the last image seen before the cut. Accordingly, if experienced drivers anticipate more than inexperienced ones, then we can expect the asymmetry between forward and backward shifts (on "same" responses) to be greater for experienced drivers than for inexperienced ones.

\section{Method}

\section{Participants}

Seventy participants took part in the experiment. They were divided into two groups on the basis of their driving experience. The "inexperienced" group was made up of 35 young adults who did not have their driver's licence (mean age: 21 years 5 months, standard deviation: 1 year 3 months). The "experienced" group contained 35 drivers (mean age: 36 years 5 months, standard deviation: 10 years 3 months) who had been driving regularly (for at least 2 hours a day) for an average of 18 years (standard deviation: 10 years 6 months). To 
make sure the participants in the experienced-driver group were "good" drivers, only drivers who had never caused a road accident and had never gotten a moving violation were selected for the study. 1

All participants had normal or corrected-to-normal vision and were naive to the purpose of the study until the experiments were over.

\section{Materials}

The monitor was located approximately $60 \mathrm{~cm}$ from the observer, and was presented in normal room illumination. All driving scenes were filmed with a digital camera. The montage of the videos was done using Pinnacle Studio Plus Version 10 software. The experiment was run on a Dell Latitude 120L portable computer. Fifteen basic videos, corresponding to 15 different places, were made. The car was always moving at a speed of 60 $\mathrm{km} / \mathrm{hr}$. In each video, a black screen lasting $250 \mathrm{~ms}$ (interstimulus interval or ISI) was inserted after 3 seconds of the video. Following this interruption, the video resumed in one of nine conditions. In the normal-resumption condition, the video resumed exactly where it had been cut, i.e., the first image after the cut was identical to the last image before the cut (hereafter called the "standard frame"). In the forward-shift conditions, the video resumed at a point corresponding to $+3 \mathrm{~m},+6 \mathrm{~m},+9 \mathrm{~m}$, or $+12 \mathrm{~m}$ past the location where the car was last seen ( $+3 \mathrm{~m}$ was the location closest to the standard frame). In the backward-shift conditions, the video resumed $-3 \mathrm{~m},-6 \mathrm{~m},-9 \mathrm{~m}$, or $-12 \mathrm{~m}$ behind the interruption point. Figure 1 gives an illustration of a standard frame, a 12-m shift forward, and a 12-m shift backward.

Insert Figure 1 here

\section{Procedure}

The experiment was run in two phases: a task-familiarization phase, followed by the experimental phase. Before the familiarization phase, the experimenter gave the participants the following instructions: "You are going to see some automobile-driving videos filmed from the driver's point of view. After a few seconds, the video will be interrupted for a short while.

\footnotetext{
1 In France, a driver's license owner is assigned merit points that are taken away every time a violation is committed. Only individuals who had never lost a point were included in the study.
} 
After the interruption, the video will resume either at exactly the same point as right before the cut, or at a different point. Your task will be to judge whether the video resumes at the same point or at a different point, and respond by pressing the green key to answer 'same' or the red key to answer 'different' ". Once they had heard the instructions, the participants performed three practice trials on three different driving scenes: one with normal resumption, one with a forward shift of $12 \mathrm{~m}$, and one with a backward shift of $12 \mathrm{~m}$. Next they performed 135 experimental trials (15 locations x 9 resumption conditions). All videos were presented in a different random order for each participant. An illustration of the procedure is given in Figure 2.

Insert Figure 2 here

\section{Results}

Three analyses were conducted. The first is an analysis of RM magnitude using PSEs; the second pertained to the percentage of "same" responses in the normal-resumption condition; the third looked at the percentage of "same" responses in the shifted-resumption conditions.

Figure 3 plots the percentage of "same" responses, by group and shift distance.

Insert Figure 3 here

\section{Analysis of RM magnitude}

The point of subjective equality (PSE) (see Jarraya, Amorim, \& Bardy, 2005) is a psychophysics measure that estimates the size of the shift a participant sees as being equal to a standard (here, the standard frame). PSE is obtained by averaging the upper differential threshold (UDT) and the lower differential threshold (LDT). For forward shifts, UDT is equal to the shift size for which participants respond 50\% "same" and 50\% "different" (i.e., it corresponds to the highest degree of uncertainty). Likewise for LDT, which pertains to backward shifts. A positive PSE reflects an anticipation bias. 
The experienced drivers' mean PSE was $1.82 \mathrm{~m}(\mathrm{SD}=1.43)$. The inexperienced drivers had a mean of $1.10 \mathrm{~m}(\mathrm{SD}=1.25)$. Each of these means was significantly different from zero $[t(34)=7.51, \mathrm{p}<.001)$, and $t(34)=5.19, \mathrm{p}<.001)$, respectively]. The difference between them was statistically significant $[\mathrm{F}(1,68)=4.99$, Mse $=1.81, \mathrm{p}<.05]$

\section{Analysis of the normal-resumption condition}

An ANOVA was conducted on the percentage of "same" responses in the normalresumption condition, as a function of the participants' level of expertise. The results indicated a significant effect of expertise $[\mathrm{F}(1,42)=4.99, \mathrm{Mse}=232.81, \mathrm{p}<.05]$. The percentage of "same" responses was significantly higher for inexperienced drivers than for experienced ones (85\% vs. $74.67 \%)$.

\section{Analysis of forward/backward shift asymmetry}

A second ANOVA was conducted on the percentage of "same" responses, with expertise level (inexperienced vs. experienced drivers) as a between-participant factor, and shift direction (forward vs. backward) and shift distance ( $3 \mathrm{~m}, 6 \mathrm{~m}, 9 \mathrm{~m}, 12 \mathrm{~m})$ as withinparticipant factors. The results did not indicate a main effect of expertise $[F(1,68)=1.15$, Mse $=1164.9]$. The shift-direction effect was significant $[F(1,68)=79.95$, Mse $=334.4$, $\mathrm{p}<.01]$ : there were more "same" responses (i.e., more errors) for forward shifts than for backward shifts, which corresponds to a forward/backward asymmetry. The results also indicated a significant effect of shift distance $[\mathrm{F}(3,204)=220.72$, Mse $=158.6, \mathrm{p}<.01]$. The greater the shift distance, the smaller the number of "same" responses. Interaction between shift-direction and shift distance was significant $[\mathrm{F}(3,204)=3.06 ;$ Mse $=136.88 ; \mathrm{p}<0.05$. The greater the shift distance, the smaller forward/backward asymmetry.

The three-way interaction between expertise, shift direction, and shift distance was significant $[\mathrm{F}(3,204)=3.16, \mathrm{Mse}=136.88, \mathrm{p}<.05]$. The planned comparisons showed that experienced drivers exhibited a stronger asymmetry than inexperienced drivers on 3-m shifts $[\mathrm{F}(1,68)=7.97, \mathrm{Mse}=188.59, \mathrm{p}<.01]$. The experienced drivers exhibited a significant forward/backward asymmetry for 12-m shifts $[\mathrm{F}(1,68)=15.04$, Mse $=195.62, \mathrm{p}<.001]$, but the inexperienced drivers did not $[\mathrm{F}(1,68)=1.86, \mathrm{Mse}=195.62, \mathrm{p}=.17]$. Figure 4 presents the forward-shift/backward-shift comparison for each shift distance.

Insert Figure 4 here 


\section{Discussion}

The results of this experiment indicated that all participants of both driving-expertise levels exhibited an RM effect. The PSEs are different from zero for both group and responses for the forward and backward shifts given by both groups of participants were asymmetrical, i.e., the error rate was significantly higher for forward shifts than for backward ones. This finding obtained with real videos corroborates those obtained with synthesized images by Thornton and Hayes (2004).

Concerning the main goal of this study, i.e., to explore the effect of domain-specific knowledge on motion anticipation, the results showed that the experienced drivers did indeed anticipate more than the inexperienced ones $\mathrm{did}^{2}$. The following findings confirmed this result. Firstly, the experienced drivers' PSE was higher than that of the inexperienced ones (with the larger group size). Secondly, when the video resumed at exactly the same place as before the cut (normal-resumption condition), experienced drivers made more errors than did inexperienced ones. Thirdly, the asymmetry obtained in the 3-m shift condition was greater for experienced drivers than for inexperienced ones. Fourthly, while for experienced drivers the asymmetry persisted for 12-m shifts, for inexperienced drivers the forward/backward asymmetry no longer existed at this shift distance.

In short, the findings of this first experiment indicate that knowledge acquired from years of driving modulates the effect of representational momentum on driving-scene judgments. Experiment 2 was aimed at determining whether this expertise effect is due to the existence of a general anticipation ability acquired with driving expertise, or whether the knowledge developed by experienced drivers is domain-specific.

${ }^{2}$ Note that in the present experiment (as in many studies on expertise), the age of the participants covaried with expertise. However, while nearly every study on age-related effects on cognition has found a decline or at least stability with aging, our older participants anticipated more than the younger ones. The anticipation advantage acquired with the development of expertise may compensate for the deleterious effects of age generally observed (e.g., Rozencwajg, Lemoine, Rolland-Grot, \& Bompard, 2005). 


\section{Experiment 2}

The purpose of this experiment was to find out whether the expertise effect observed in Experiment 1 can be generalized to other dynamic environments that are far-removed from the one in which the expert knowledge was acquired. Participants assigned to two groups on the basis of their driving experience conducted an RM task on dynamic scenes that did not pertain to automobile driving. The hypothesis tested was that anticipation processes developed in a given domain are not transferred to other domains. With this hypothesis, we can expect no differences between experienced and inexperienced drivers in terms of movement anticipation.

\section{Method}

\section{Participants}

Forty-four participants of Experiment 1 took also part in Experiment 2, namely, 20 young adults who did not have their driver's license and 24 experienced drivers.

\section{Materials}

The monitor was located approximately $60 \mathrm{~cm}$ from the observer, and was presented in normal room illumination. The material consisted of two types of videos, ones presenting a natural scene and ones presenting an artificial scene (see Figure 5).

In the natural-scene videos - "natural" in the sense that they showed a human action filmed by a stationary camera - a person was seen running from left to right ${ }^{3}$ across the screen. The person was moving at a constant speed $(5.5 \%)$ in a relatively homogeneous environment (a quarry with light-colored soil). Two seconds after the beginning of the video, a black screen was displayed for $250 \mathrm{~ms}$. After the interruption, the video resumed in one of the same nine conditions as in Experiment 1 (normal-resumption condition, forward-shift

3 Given that we did not set forth any hypotheses about the potential effect of the direction of motion, we chose the direction that seemed the most likely to produce an RM effect. Halpern and Kelly (1993) reported that left-to-right motion gave rise to a greater shift. Note, however, that Hubbard (1990, 1995), Hubbard and Bharucha (1988), and Cooper and Munger (1993) did not find such differences. 
conditions, and backward-shift conditions). In both shift conditions, there were four shift distances (meters travelled by the person in the video): $0.25 \mathrm{~m}, 0.5 \mathrm{~m}, 0.75 \mathrm{~m}, 1 \mathrm{~m}$.

The artificial scene showed an animated (24fps) black square moving from left to right $(5.5 \%)$ across the screen against a plain, light-colored background. As above, after two seconds of animation, a black screen appeared for $250 \mathrm{~ms}$ before the rest of the video was shown, in one of the nine resumption conditions: normal-resumption condition, forward-shift conditions, and backward-shift conditions, the last two of which had four shift distances each (expressed in number of images): 4 images, 8 images, 12 images, and 16 images. This made nine videos per type of scene.

Insert Figure 5 here

\section{Procedure}

Forty-four participants who had taken part in Experiment 1 went directly on to this experiment after a short break of a few minutes. The procedure was the same as in Experiment 1. All participants performed two blocks of trials in succession: one showing natural scenes and one showing artificial scenes (the testing order for the two blocks was counterbalanced across participants). Within each block, the items were presented in random order. Items in the natural-scene block were repeated six times; those in the artificial-scene block were repeated four times. This made for a total of 54 natural scenes and 36 artificial scenes. The participant's task was the same as in Experiment 1.

\section{Results}

\section{Artificial scenes (moving square)}

Figure 6 presents all results obtained for the artificial scenes.

Insert Figure 6 here 
Analysis of RM magnitude. The mean PSE of the experienced drivers was 3.65 images (i.e, $15.2 \mathrm{~ms})(\mathrm{SD}=2.93)$; it was 2.63 images (i.e, $10.95 \mathrm{~ms})(\mathrm{SD}=2.35)$ for the inexperienced drivers. Each of these values is significantly different from zero $[t(23)=6.11$, $\mathrm{p}<.001$, and $t(19)=4.99, \mathrm{p}<.001$, respectively]. The difference between these two means was nonsignificant $[\mathrm{F}(1,42)=1.58, \mathrm{Mse}=7.22, \mathrm{p}=.21]$.

Analysis of normal-resumption condition. The ANOVA on "same" responses in the normal-resumption condition did not yield a significant effect $[\mathrm{F}(1,42)=.94$, Mse $=877.7$, $\mathrm{p}=.33]$. The percentage of "same" responses given by experienced drivers (59.49\%) was not statistically different from that of inexperienced drivers $(66.25 \%)$.

Analysis of forward/backward shift asymmetry. The ANOVA on the artificial scenes, with expertise as a between-group factor and shift direction and distance as within-group factors, did not yield an expertise effect $[\mathrm{F}(1,42)=.0001$, Mse $=952.9, \mathrm{p}=.99]$. The shiftdirection factor had a significant effect $[\mathrm{F}(1,42)=90.3$, Mse $=1265.89, \mathrm{p}<.01]$, and so did the shift-distance factor $[\mathrm{F}(3,126)=79.47, \mathrm{Mse}=578.98, \mathrm{p}<.01]$. There were no significant interactions.

\section{Natural scenes (person running)}

Figure 7 presents all results obtained for the natural scenes.

Insert Figure 7 here

Analysis of RM magnitude. The mean PSE of the experienced drivers was $0.33 \mathrm{~m}$ $(\mathrm{SD}=0.14)$; it was $0.38 \mathrm{~m}(\mathrm{SD}=0.15)$ for the inexperienced drivers. Both of these values are significantly different from zero $[t(23)=11.73, \mathrm{p}<.001$, and $t(19)=10.95, \mathrm{p}<.001$, respectively]. The difference between these two means was nonsignificant $[\mathrm{F}(1,42)<1]$.

Analysis of normal-resumption condition. The ANOVA conducted on "same" responses for natural scenes in the normal-resumption condition did not indicate a significant effect $[\mathrm{F}(1,42)=1.23, \mathrm{Mse}=489.01, \mathrm{p}=.27]$. The percentage of "same" responses given by experienced drivers $(64.23 \%)$ did not differ significantly from that of inexperienced drivers $(71.66 \%)$.

Analysis of forward/backward shift asymmetry. The ANOVA on the percentage of "same" responses for natural scenes, with expertise as a between-group factor and shift 
direction and distance as within-group factors, revealed a main effect of expertise [F (1, $42)=5.13$, Mse $=603.98, \mathrm{p}<0.05]$. On average, inexperienced drivers responded "same" a little more often than experienced drivers did. The shift-direction effect was significant [F (1, $42)=270.31$, Mse $=708.08 \mathrm{p}<.01]$, as was the shift-distance effect $[\mathrm{F}(1,126)=62.3$, Mse $=328.63, \mathrm{p}<.01]$. The interaction between shift direction and shift distance was also significant $[\mathrm{F}(3,126)=12.11$, Mse $=244.91, \mathrm{p}<.01]$. The three-way interaction between expertise, shift direction, and shift distance was nonsignificant $[\mathrm{F}(3,126)=.38$, Mse $=244.91, \mathrm{p}=.76]$.

\section{Discussion}

The results of Experiment 2 showed that for both natural scenes and artificial scenes, all participants exhibited an RM effect. As a whole, the participants made judgment errors in the normal-resumption condition, their PSE values were significantly greater than zero, and their forward-shift and backward-shift responses were asymmetrical (i.e., there were more mistakes on forward shifts than on backward ones). These results thus provide further evidence of an RM effect in dynamic situations. Unlike Experiment 1, however, there was no anticipation difference between the two groups of participants (experienced or inexperienced drivers).

Note that an unexpected result was obtained in this study for natural scenes: expertise had a main effect. No matter where the video resumed, the inexperienced drivers answered "same" slightly more often than the experienced drivers did, although the shape of the response distributions was similar for the two groups. Such an effect was not found in Experiment 1, nor was it observed for the artificial scenes in Experiment 2. This finding (for which we have no explanation) is probably not linked to the processes underlying the RM effect - which was the focus of the present study - so it will not receive further comments here. Ruppel, et al (2009) found similar results and discuss how the height of the distribution does not reflect representational momentum, but the asymmetry of the distribution does reflect representational momentum.

The main finding of Experiment 2 is that knowledge acquired in a specific domain (here, automobile driving), which led to RM modulation with experience, was not transferred to dissimilar domains. Note that an anticipation difference could have been obtained because of the age difference between the two groups (mean age 20 for the inexperienced drivers vs. 38 for the experienced drivers). DeLucia and Mather (2006) showed that age tended to slow 
down the extrapolation of motion. However, in their study, the older participants (mean age 58) were much older than the experienced drivers in our study. Furthermore, age-linked cognitive declines have mainly been demonstrated in individuals age 50 or older (Kausler, 1991; Salthouse, 1982).

\section{Correlations between Experiments 1 and 2}

Correlations were calculated in order to relate the participants' performance of the 44 participants which took part in both experiments on the driving scenes in Experiment 1 and on of the two types of scenes in Experiment 2. Correlations between natural-scene performance and artificial-scene performance in Experiment 2 were also computed. This gave us six correlations (three for the inexperienced group and three for the experienced group). The correlations were calculated on the PSE values.

For the novices, nonsignificant correlations were obtained between the driving-scene data and the natural-scene data $(r=.08, \mathrm{p}>.05)$, between the driving-scene data and the artificial-scene data $(r=-.25, \mathrm{p}>.05)$, and between the natural-scene data and the artificialscene data $(\mathrm{r}=.09, \mathrm{p}>.05)$. For the experienced drivers, nonsignificant correlations were also obtained between the driving scenes and the natural scenes $(r=-.12, p>.05)$, between the driving scenes and the artificial scenes $(r=-.04, p>.05)$, and between the natural scenes and the artificial scenes $(r=.18, p>.05)$. The fact that no correlations were found between these different types of scenes, for either group of participants, argues in favor of the presence of $\mathrm{RM}$ components specific to the scenes presented.

\section{General discussion}

This research had two objectives: find out if the RM effect is sensitive to expertise in the specific domain of automobile driving, and determine whether knowledge acquired with expertise is transferable to other types of scenes that are not from the observer's domain of expertise.

Our results showed, first of all, that for the natural dynamic scenes we used, all participants exhibited an RM effect. They extend Thornton and Hayes's (2004) findings (obtained using synthesized automobile-driving scenes) to real scenes that were actually filmed. They also add to the range of situations in which the RM effect can be observed. The $\mathrm{RM}$ effect is found not only in situations where participants are watching a scene containing a 
moving object or objects, but also in situations where the participant is an integral part of the movement (Munger, Owens, \& Conway, 2005; DeLucia \& Maldia, 2006). This study placed the observer in the driver's position in a car moving at a constant speed, so the observer saw an ever-changing visual scene. In such situations, an RM effect is always observed.

Experiment 1 comparing experienced drivers and persons who had never driven showed that some RM components can be modulated by expertise. Firstly, the experienced drivers had a higher PSE. Secondly, the experienced drivers made more errors when the video resumed at exactly the same point as before the cut. Thirdly, unlike the inexperienced drivers, the experienced ones exhibited more asymmetry for smaller shifts, and an asymmetry that persisted up to $12 \mathrm{~m}$. Accordingly, in the road scenes used, the more experienced the drivers were, the greater the amplitude of the representational momentum. Thus, expert knowledge of some kind had an impact on anticipation processes in dynamic-scene processing, provided the scenes are from the observer's domain of expertise. These results extend earlier findings from the few studies demonstrating RM modulation by the observer's domain-specific conceptual knowledge of the moving object (Vinson \& Reed, 2002). They also show, as noted in certain models of expert memory (for a review, see Gobet, Lane, Croker, Cheng, Oliver, \& Pine, 2001), that expert perception of scenes differs from that of novices right from the perceptual encoding phase.

The results of Experiment 2 showed that RM modulation as a function of observer expertise in a specific domain (automobile driving) was not generalized to other domains. For the visual scenes studied, i.e., a moving square on a plain background and a person running in a natural environment, the experienced drivers exhibited the same RM effect as the other participants, so there was no transfer of expertise. A question that arises here concerning the wide variety of situations in which an RM effect is observed (for a review, see Hubbard, $2005)$ is whether these effects are rooted in a single, general skill or in multiple, specific skills. Our results indicate that at least part of experts' knowledge is domain-specific. The absence of correlations between each group's performance on the three different types of scenes used here suggests that certain processes underlying RM are task-specific.

The two studies reported here point out some properties of representational momentum. Although the fact of having found an RM effect in a wide variety of situations suggests that at least one of the components of RM is generic, the present results seem to show that RM might be composed of a set of both general and domain-specific processes. Our research showed that expertise in automobile driving can modulate RM in road-scene perception: the cognitive characteristics of the observer can change the magnitude of the RM effect. The next question to be answered concerns what specific anticipation processes are 
implemented to analyze these scenes as a function of their characteristics, including whether the observer is moving along with the scene, and whether he/she is making decisions about what action to take next. In this vein, the research conducted by Jordan and Hunsinger (2008) demonstrated that action control can have an impact on perception. These authors compared the estimated vanishing points given by participants trained in advance to control the motion of a stimulus moving horizontally on a screen (using two knobs for increasing or decreasing the speed of the stimulus) to those of participants who had never had the opportunity to control the motion of the stimulus, and showed that the trained participants exhibited a greater forward displacement (FD). The hypothesis tested was that experience acquired by "observers-actors" about the action-effect coupling on distal events has an impact on perception, even in situations where the movement of the stimulus can no longer be controlled. The theoretical framework for interpreting this effect was the Theory of Event Coding or TEC (Hommel, Müssler, Aschersleben, \& Prinz, 2001). Neurophysiological data support this interpretation. Decety (2002), for example, showed that the brain areas activated when a person is planning an action are also activated when the person is watching that action. In this context, the learning of action-effect anticipation patterns is thought to influence FD magnitude, in such a way that the best possible fit between the actions and the dynamic environment presented is achieved.

Jordan and Hunsinger (2008) themselves drew an analogy between the experimental situation they used and that of automobile drivers who are acting as simple passengers. They hypothesized that driving or riding in an automobile modifies the observer's perception of driving situations they have experienced. This is exactly what we observed by comparing experienced drivers and inexperienced drivers. It therefore seemed relevant to try to operationalize the TEC in situations of road-scene perception. Relevant, but not trivial insofar as the road scenes processed by our participants were much more complex than the experimental situations used by Jordan and Hunsinger (2008); and they were all different. Thus, the actions to be carried out always had to be modulated by environment-specific characteristics, and participants with expertise in a specific domain would be more sensitive to the relevant environment-specific characteristics in that domain. Moreover, action schemas take months or years to be acquired, not a few dozen trials. Attempting to operationalize TEC in this context nevertheless seems to us to be a promising avenue for identifying the more or less specific patterns of action-effect anticipation that are acquired with experience.

Even in this framework, it would be worthwhile to gain deeper insight into the relationships between attention and RM. (see, Hayes \& Freyd, 2002; Kerzel, 2003; Munger \& Owens, 2004). Pearson and Schaefer (2005) showed that participants' involvement in a 
change-blindness task on photographs of road scenes modified their performance. Participants in the so-called cognitive-engagement condition were told that the results of the study might be of diagnostic value in identifying people in need of remedial driver training. This sole piece of information gave rise to significant differences between the performance of simple observers and observers who were more involved in the automobile-driving situation. Not only the changes occurring in the main areas of interest in the scene, but also changes that were in marginally interesting areas but were nonetheless relevant to automobile driving, were detected better by the more-involved participants, due solely to the instructions they had been given. These findings are consistent with views of attention that allow for increases in attentional resources and a flexible distribution of attention (Kahneman, 1973). The results we obtained here by comparing observers who were "expert" automobile drivers and others who were "novices" can be interpreted in this framework. Compared to novices, experts may feel more involved in the action and so may allocate their attention differently (see Reingold, Charness, Pomplun, \& Stampe, 2001 and Reingold, Charness, Schultetus, \& Stampe,, 2001 for examples on chess experts). On this point, an analysis of drivers' eye movements could provide some interesting information. Whatever the case may be, a key topic that remains to be studied concerns the relative contributions of exogenous and endogenous factors in the mechanisms of attention allocation and decision-making about what actions will lead to the most optimal fit to the dynamic situations encountered.

\section{References}

Bertamini, M. (1993). Memory for position and dynamic representations. Memory \& Cognition, 21, 449-457.

Chase, W.G., \& Simon, H.A. (1973). Perception in chess. Cognitive Psychology, 4, 55-81.

Courtney, J.R., \& Hubbard, T.L. (2008). Spatial memory and explicit knowledge: an effect of instruction on representational momentum. Quarterly Journal of Experimental Psychologyl, 61(12), 1778-1784.

Cooper, L.A., \& Munger, M.P. (1993). Extrapolations and remembering positions along cognitive trajectories: Uses and limitations of analogies to physical momentum. In N. Eilan, R. McCarthy, \& B. Brewer (Eds.), Spatial representation: Problems in philosophy and psychology (pp. 112-131). Cambridge, MA: Blackwell.

Decety, J. (2002). Is there such a thing as functional equivalence between imagined, observed, and executed action?. In A. Meltzoff \& W. Prinz. 
DeLucia, P.R., \& Maldia, M.M. (2006). Visual memory for moving scenes. Quarterly Journal of Experimental Psychology, 59, 340-360.

DeLucia, P.R., \& Mather, R.D. (2006). Motion extrapolation of car-following scenes in younger and older drivers. Human Factors, 48, 666-674.

Didierjean, A., \& Gobet, F. (2008). Sherlock Holmes - An expert's view of expertise. British Journal of Psychology, 99, 109-125.

Didierjean, A., \& Marmèche E. (2005). Anticipatory representation of visual basketball scenes by novice and expert players. Visual Cognition, 12, 265-283.

Doane, S.M., Sohn, Y. W., \& Jodlowski, M. T. (2004). Pilot ability to anticipate the consequences of flight actions as a function of expertise. Human Factors, 46, 92-103.

Ericsson, K.A. (1985). Memory skill. Canadian Journal of Psychology, 39, 188-231.

Ericsson, K.A., \& Kintsch, W. (1995). Long-term working memory. Psychological Review, $102,211-245$.

Ferrari, V., Didierjean, A., \& Marmèche, E. (2006). Dynamic perception in chess. Quarterly Journal of Experimental Psychology, 59, 397-410.

Finke, R.A., Freyd, J.J., \& Shyi, G.C. (1986). Implied velocity and acceleration induce transformations of visual memory. Journal of Experimental Psychology: General, 115, 175-188.

Finke, R.A., \& Shyi, G.C. (1988). Mental extrapolation and representational momentum for complex implied motions. Journal of Experimental Psychology: Learning, Memory, and Cognition, 14(1), 112-120.

Freyd, J.J. (1983). The mental representation of movement when static stimuli are viewed. Perception \& Psychophysics, 33, 575-581.

Freyd, J.J., \& Finke, R.A. (1984). Representational momentum. Journal of Experimental Psychology: Learning, Memory, and Cognition, 10, 126-132.

Freyd, J.J., \& Finke, R.A. (1985). A velocity effect of representational momentum. Bulletin of the Psychonomic Society, 23, 443-446.

Freyd, J.J., Pantzer, T.M., \& Cheng, J.L. (1988). Representing statics as forces in equilibrium. Journal of Experimental Psychology: General, 117, 395-407.

Gauthier, I., Williams, P., Tarr, M.J., \& Tanaka, J. (1998). Training 'greeble' experts: a framework for studying expert object recognition processes. Vision Research, 38, 2401-2428.

Gobet, F., Lane, P.C.R., Croker, S., Cheng, P.C.H., Jones, G., Oliver, I., \& Pine, J.M. (2001). Chunking mechanisms in human learning. Trends in Cognitive Sciences, 5, 236-243. 
Halpern, A.R., \& Kelly, M.H. (1993). Memory biases in left versus right implied motion. Journal of Experimental Psychology: Learning, Memory, and Cognition, 19, 471-484.

Hatano, G., \& Osawa, K. (1983). Digit memory of grand experts in abacus-derived mental calculation. Cognition, 15, 95-110.

Hayes, A.E., \& Freyd, J.J. (2002). Representational momentum when attention is divided. Visual Cognition, 9, 8-27.

Hommel, B., Musseler, J., Aschersleben, G., \& Prinz, W. (2001). The Theory of Event Coding (TEC): a framework for perception and action planning. Behavioral Brain Sciences, 24(5), 849-878; discussion 878-937.

Hubbard, T.L. (1990). Cognitive representation of linear motion: possible direction and gravity effects in judged displacement. Memory \& Cognition, 18, 299-309.

Hubbard, T.L. (1995). Environmental invariants in the representation of motion: Implied dynamics and representational momentum, gravity, friction, and centripetal forces. Psychonomic Bulletin \& Review, 2, 322-338.

Hubbard, T.L. (1996). Displacement in depth: representational momentum and boundary extension. Psychological Research, 59(1), 33-47.

Hubbard, T.L. (1998). Some effects of representational friction, target size, and memory averaging on memory for vertically moving targets. Canadian Journal of Experimental Psychology, 52, 44-49.

Hubbard, T.L. (2005). Representational momentum and related displacements in spatial memory: A review of the findings. Psychonomic Bulletin \& Review, 12, 822-851.

Hubbard, T.L., \& Bharucha, J.J. (1988). Judged displacement in apparent vertical and horizontal motion. Perception \& Psychophysics, 44, 211-221.

Jarraya, M., Amorim, M. A., \& Bardy, B.G. (2005). Optical flow and viewpoint change modulate the perception and memorization of complex motion. Perception \& Psychophysics, 67(6), 951-961.

Jordan, J.S., \& Hunsinger, M. (2008). Learned patterns of action-effect anticipation contribute to the spatial displacement of continuously moving stimuli. Journal of Experimental Psychology: Human Perception and Performance, 34(1), 113-124.

Kahneman, D. (1973). Attention and effort. Englewood Cliffs, NJ: Prentice Hall.

Kausler, D. H. (1991). Experimental Psychology, Cognition, and Human Aging. New York: Springer-Verlag.

Kelly, M.H., \& Freyd, J.J. (1987) Exploration of representational momentum. Cognitive Psychology, 19, 369-401. 
Kerzel, D. (2003). Attention maintains mental extrapolation of target position: irrelevant distractors eliminate forward displacement after implied motion. Cognition, 88(1), 109-131.

Kozhevnikov, M. \& Hegarty, M. (2001). Impetus beliefs as default heuristics: Dissociation between explicit and implicit knowledge about motion. Psychonomic Bulletin \& Review, 8, 439-453.

Munger, M.P., Solberg, J.L., Horrocks, K.K., \& Preston, A.S. (1999). Representational momentum for rotations in depth: effects of shadings and axis. Journal of Experimental Psychology: Learning, Memory, and Cognition, 25, 157-171.

Munger, M.P. \& Owens, T.R. (2004). Representational momentum and the flash-lag effect. Visual Cognition, 11, 81-103.

Munger, M.P., Owens, T.R. \& Conway, J.E. (2005). Are boundary extension and representational momentum related? Visual Cognition, 12, 1041-1056.

Nagaï, M., \& Yagi, A. (2001). The pointedness effect on representational momentum. Memory \& Cognition, 29, 91-99.

Pearson, P.M., \& Schaefer, E.G. (2005). Toupee or not toupee? The role of instructional set, centrality, and relevance in change blindness. Visual Cognition, 12(8), 1528-1543.

Reed, C.L., \& Vinson, N.G. (1996) Conceptual effect on representational momentum. Journal of Experimental Psychology: Human Perception and Performance, 2, 839-850.

Reingold, E.M., Charness, N., Pomplun, M., \& Stampe, D.M. (2001). Visual span in expert chess players: evidence from eye movements. Psychological Science, 12(1), 48-55.

Reingold, E.M., Charness, N., Schultetus, R.S., \& Stampe, D.M. (2001). Perceptual automaticity in expert chess players: parallel encoding of chess relations. Psychonomic Bulletin \& Review, 8(3), 504-510.

Rozencwajg, P., Lemoine, C., Rolland-Grot, M., Bompard, A. (2005). Combined effects of age and job experience on spatial abilities: the case of air-traffic controllers. Psychologie du Travail et des Organisations, 11, 47-57.

Ruppel, S.E., Fleming, C.N., \& Hubbard, T.L. (2009). Representational momentum is not (totally) impervious to error feedback. Canadian Journal of Experimental Psychology, 63(1), 49-58.

Salthouse, T.A. (1982). Adult cognition: An experimental psychology of human aging. New York: Springer-Verlag.

Tanaka, J.W., Curran, T., \& Sheinberg, D.L. (2005). The training and transfer of real-world perceptual expertise. Psychological Science, 16, 145-151. 
Thornton, I.M., \& Hayes, A.E. (2004). Anticipating action in complex scenes. Visual Cognition, 11, 341-370.

Unterrainer, J.M., Kaller, C.P., Halsband, U., \& Rahm, B. (2006). Planning abilities and chess: a comparison of chess and non-chess players on the Tower of London task. British Journal of Psychology, 97, 299-311.

Vinson, N.G., \& Reed, C.L. (2002). Sources of object-specific effects in representational momentum. Visual Cognition, 9, 41-65. 


\section{Figure Captions}

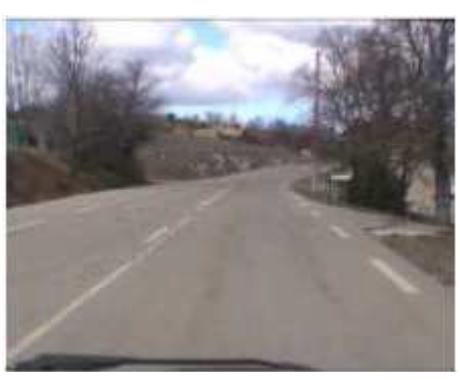

12 meters backward

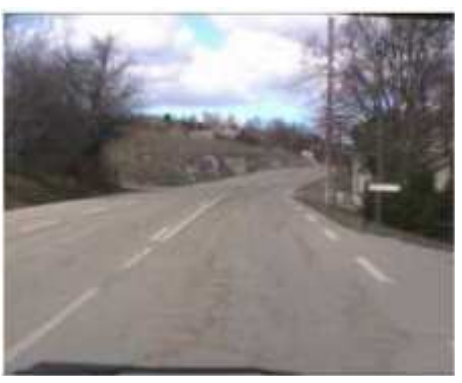

Standard frame

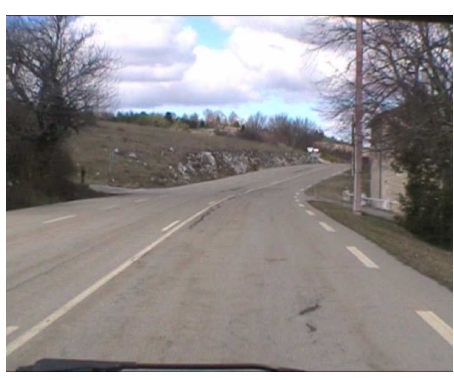

12 meters forward

Figure 1. Example of the material used in Experiment 1. The standard frame (in the middle) was the last image seen before the cut. The video resumed after a backward shift of $12 \mathrm{~m}$ or a forward shift of $12 \mathrm{~m}$.

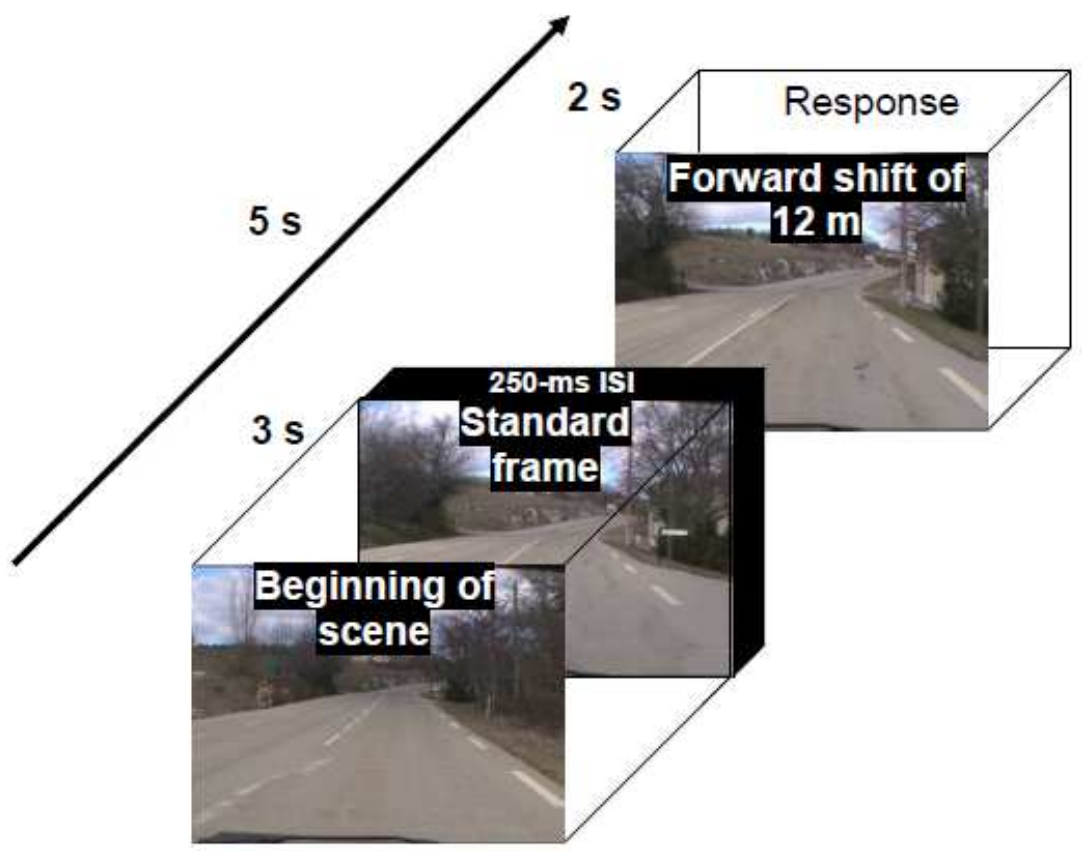

Figure 2. Illustration of the procedure. Each video was viewed for 3 seconds before the 250ms cut. The scene resumed at the point where the cut was made, after a forward shift, or after a backward shift. Top: example of a 12-m shift forward. 


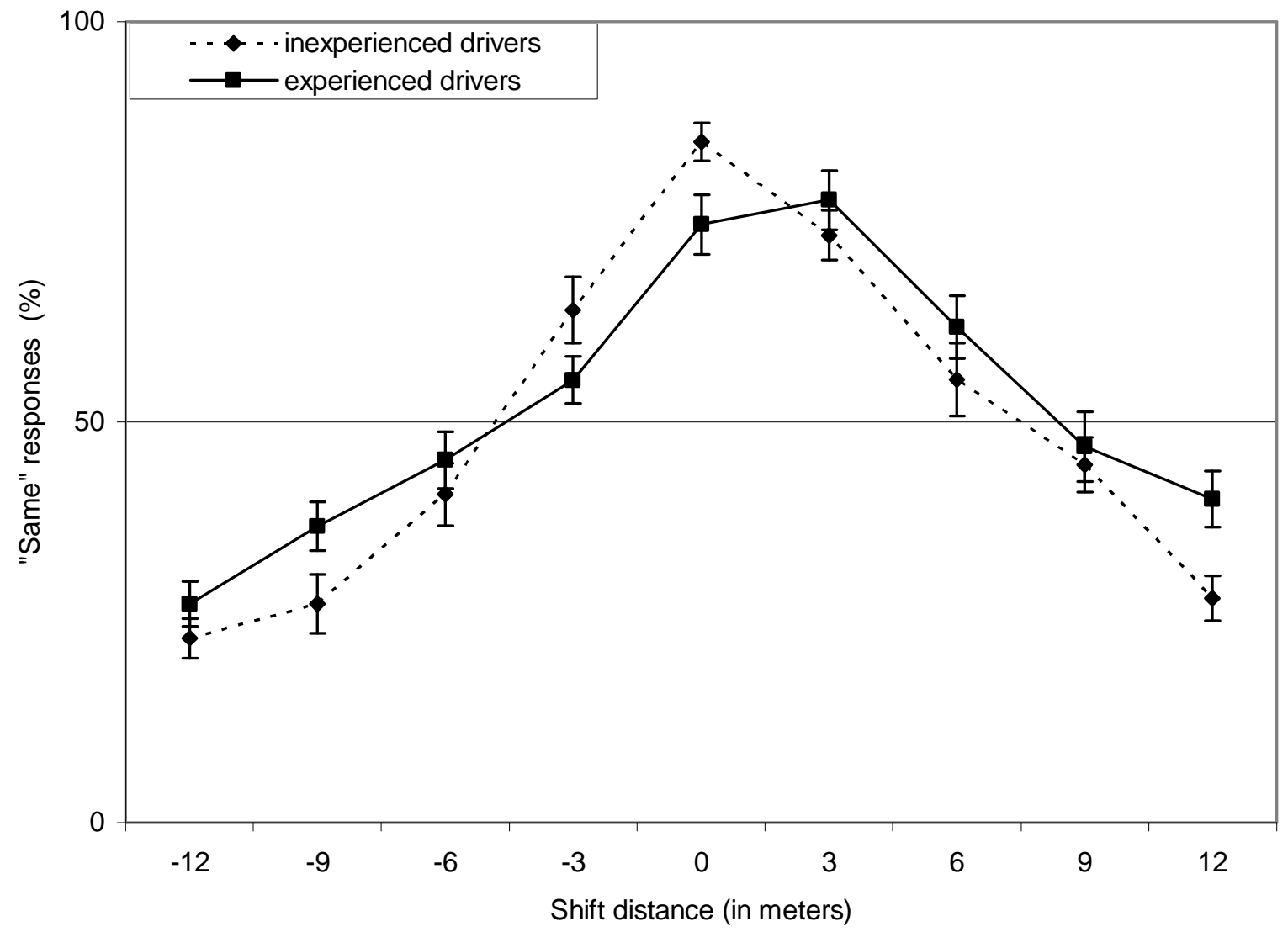

Figure 3. Percentage of "same" responses, by expertise level, shift direction, and shift distance (error bars are standard error). 


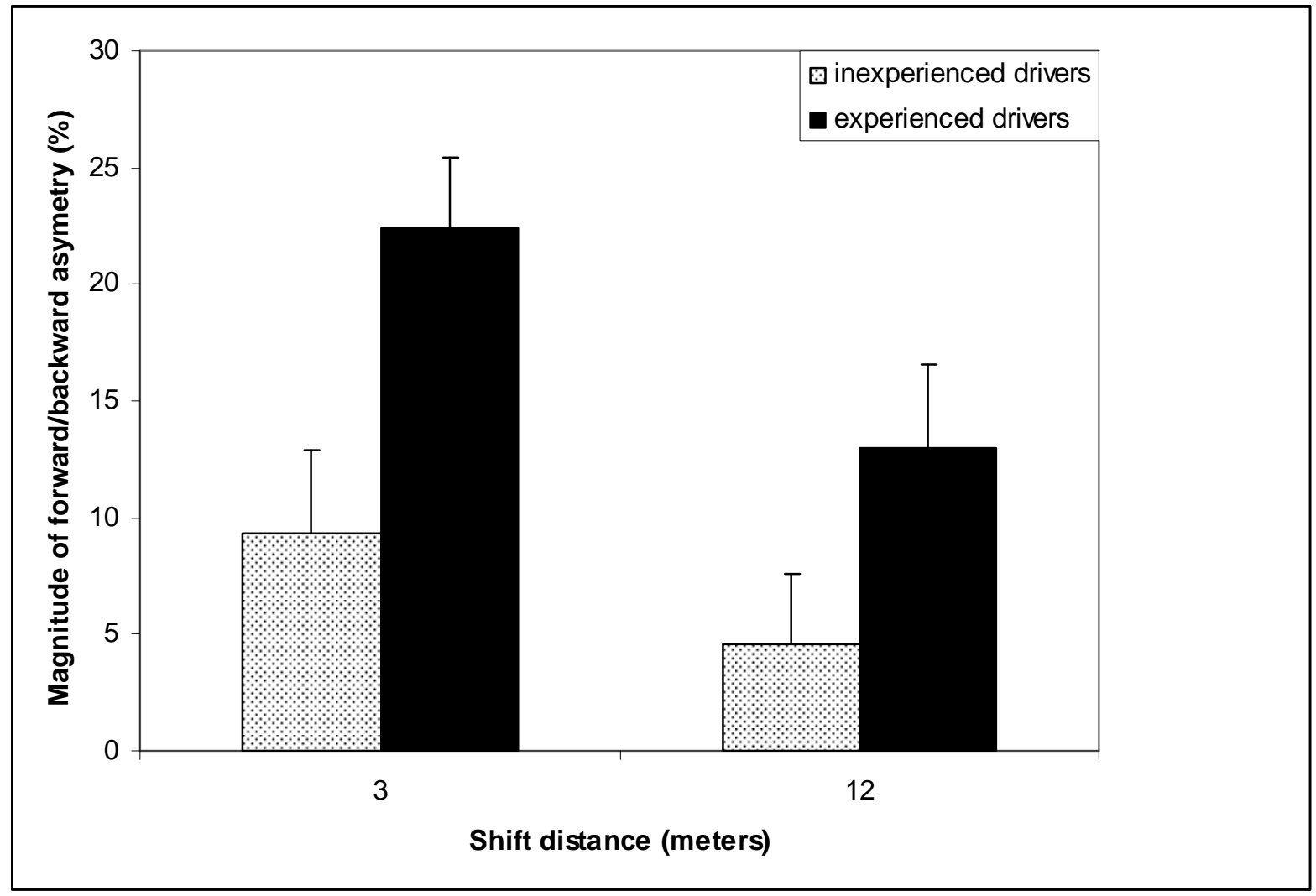

Figure 4. Differences between the percentage of "same" responses on forward and backward shifts, by shift distance and participant group (inexperienced and experienced). Error bars are standard error.
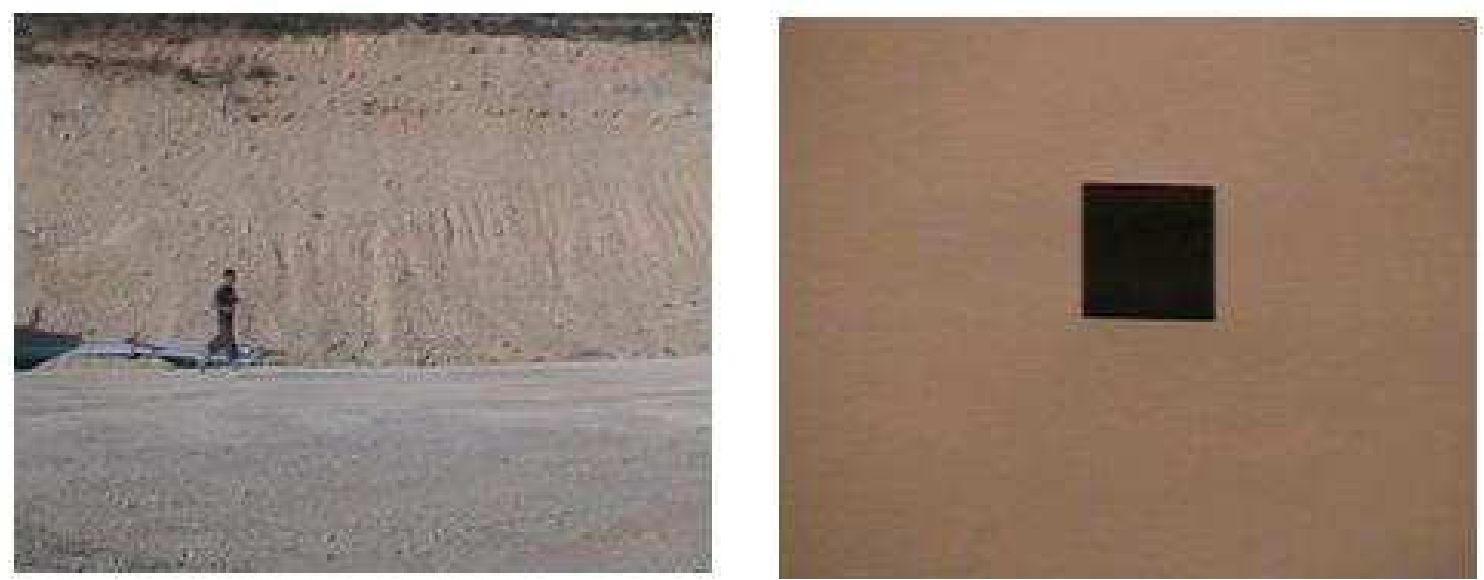

Figure 5. Examples of the material used in Experiment 2. Left: the image is a screen print of a video showing a person running from left to right. Right: the image is a screen print of an animated square moving from left to right. 


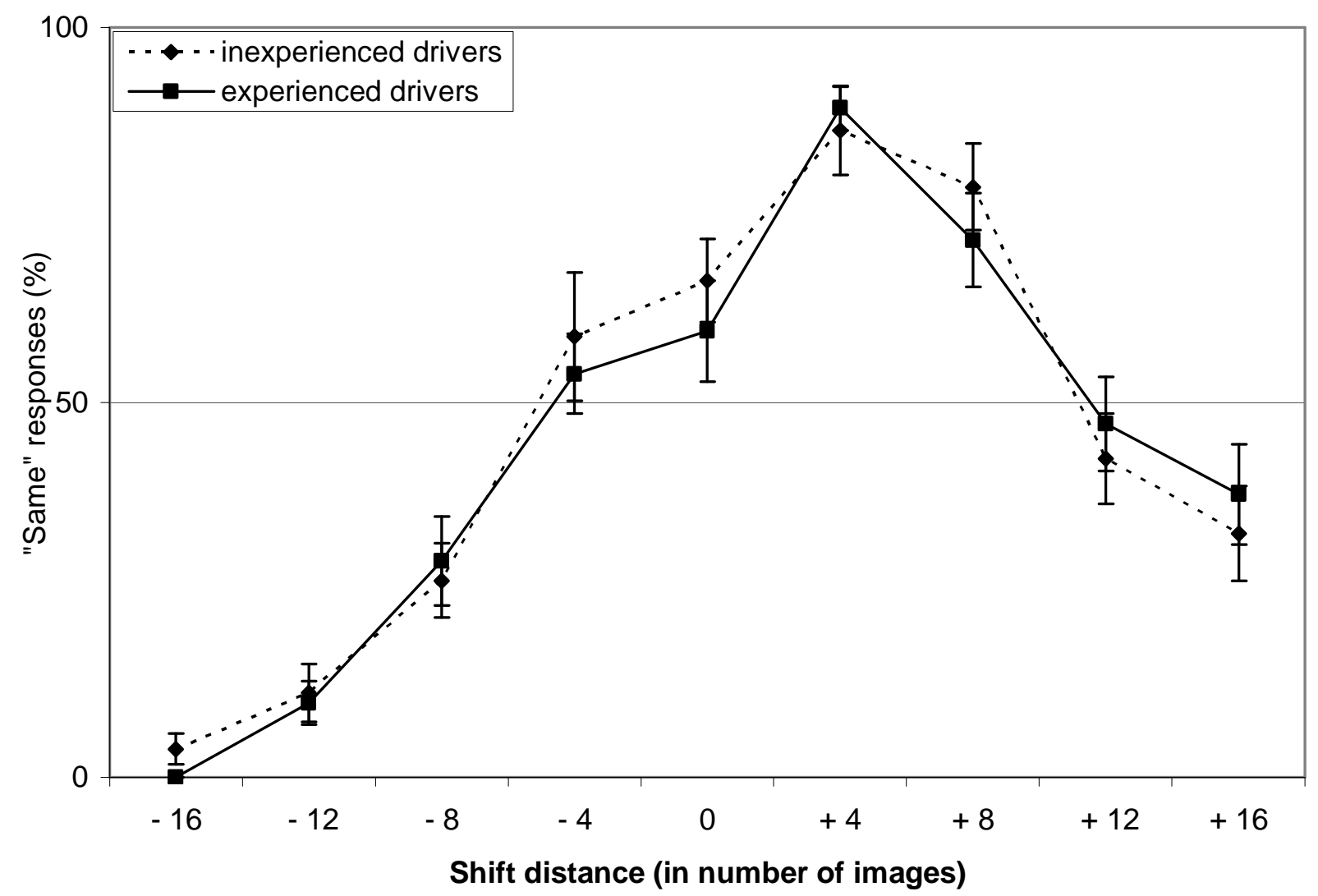

Figure 6. Percentage of "same" responses for artificial scenes, by driving experience, shift direction, and shift distance (error bars are standard error). 


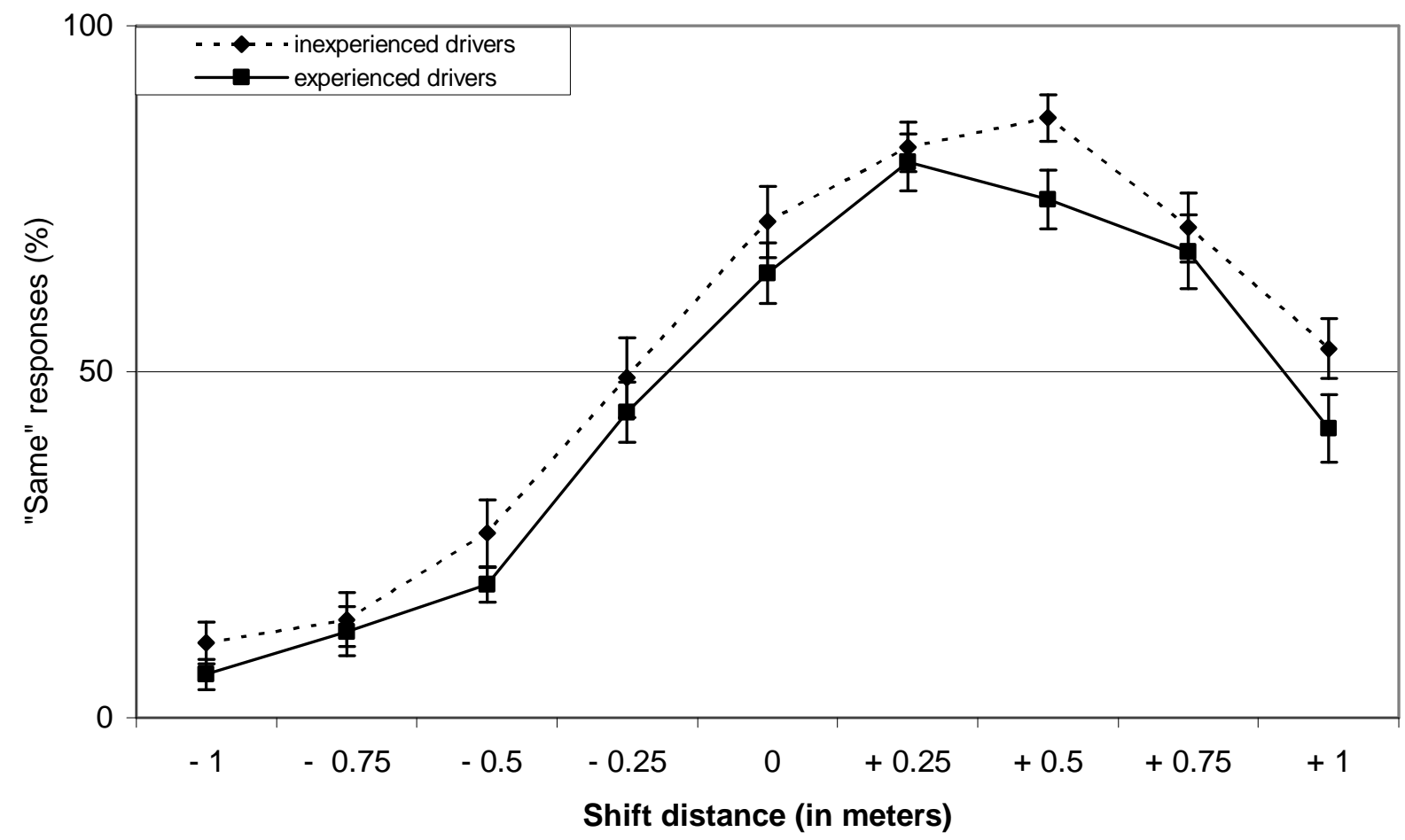

Figure 7. Percentage of "same" responses for natural scenes, by driving experience, shift direction, and shift distance (error bars are standard error). 